Article - Agriculture, Agribusiness and Biotechnology

\title{
Enhanced Production Of Streptokinase By Chemical Mutagenesis Of Streptococcus agalactiae EBL-20
}

Arooj Arshad ${ }^{1 *}$

https://orcid.org/0000-0003-0562-9484

Muhammad Anjum Zia ${ }^{1}$

http://orcid.org/0000-0003-2733-576X

Muhammad Asghar ${ }^{1}$

http://orcid.org/0000-0003-2920-2178

Faiz Ahmad Joyia ${ }^{2}$

http://orcid.org/0000-0002-9540-6972

${ }^{1}$ Department of Biochemistry, University of Agriculture, Faisalabad; ${ }^{2}$ Centre of Agricultural Biochemistry and Biotechnology, University of Agriculture, Faisalabad.

Received: 2017.12.24; Accepted: 2018.11.28.

\section{HIGHLIGHTS}

- Streptokinase isolated from Streptococcus agalactiae is an active thrombolytic agent

- Ethyl methanesulfonate is an effective mutagenic agent for Streptococcus spp.

- Response surface methodology resulted in 1.92-fold increased streptokinase activity

Abstract: Streptokinase (SK) is an enzyme that is used for the treatment of cardiovascular diseases. The current study focused on the enhanced production of SK by inducing mutation in Streptococcus agalactiae EBL-20 and optimization of medium components and culture conditions for the maximum growth of mutant derived strain. S. agalactiae EBL-32 was selected as a potent mutant after exposure of S. agalactiae EBL-20 to EMS for 180 minutes. SK activity obtained from mutant derived strain was found to be 1.6 fold higher as compared to the activity achieved by wild strain. Nutritional requirements of the mutated strain were optimized by single factor analysis method suggesting glucose as the optimum carbon source; yeast extract and peptone as a suitable nitrogen sources and corn steep liquor (CSL) as an appropriate substrate for the maximum SK production. The culture conditions determined by response surface methodology (RSM) suggested that a temperature value of $37.5^{\circ} \mathrm{C}$ and $\mathrm{pH} 7$ of the fermentation medium with $2.50 \mathrm{~mL}$ inoculum size for 36 hours of incubation was optimum for maximum yield of SK. Hence the optimization studies resulted 
into 1.92 fold increase in the yield of SK suggesting the new isolate suitable for commercial scale production of SK.

Keywords: Ethyl methanesulfonate, fermentation, response surface methodology, Streptokinase

\section{INTRODUCTION}

Cardiovascular diseases (CVDs) and their outcomes are a major cause of mortality worldwide. Complications associated with cardiovascular disorders are due to imbalance in the maintenance of homeostasis between the development of clot and its breakdown [1, 2]. Among these diseases, thrombosis is most common within the elderly population which is due to obstruction of blood circulation caused by blood clots [3]. A variety of thrombolytic agents with varied pharmacodynamic and pharmacokinetic properties are available for treating diseases like stroke, acute myocardial infarction (AMI), deep vein thrombosis (DVT) and pulmonary embolism. Streptokinase (SK) is one of those agents that are frequently used to treat such type of complications [4] mainly in world's poor health care systems due to its low cost [5].

SK (EC 3.4.99.22) is the first FDA approved thrombolytic drug emerging as a highly potential thrombolytic agent for treating different cardiovascular disorders [6]. It is an extracellular protein of $47 \mathrm{kDa}$ which is composed of 414 amino acids. The isoelectric $\mathrm{pH}$ of the protein is 4.7 showing its best activity at $\mathrm{pH}$ range of 7.0 to 7.5 [7, 8]. Activation of plasminogen by fibrinolytic drugs is used to treat blood clots. Administration of SK in cases of early stage thrombosis can avert heart muscle impairments [9]. SK acts in a fibrin independent manner and binds with both the circulatory and thrombus-bound plasminogen in a stoichiometric 1:1 ratio to convert plasminogen into plasmin. Plasmin is the active fibrinolytic constituent of the blood circulatory system which neutralizes the mesh of fibrin in blood clots leading to dissolution of thrombus [9]. SK possesses high affinity for plasminogen and has a long half-life as compared to other plasminogen activators [11].

It has been demonstrated that $\beta$ hemolytic Streptococcus of Lancefield group A, C and $G$ produces the enzyme streptokinase [12-14]. Moreover, Group B Streptococcus (S. agalactiae) has also recently been reported to produce SK [15]. Different strategies have been introduced to enhance the production of streptokinase. Strain improvement studies have been performed for inducing modifications in Streptococcus spp. resulting into high production of SK by submerged and liquid state fermentation. Ultraviolet (UV) and gamma radiations have been successfully used for physical mutagenesis; whereas, N-methyl-N'nitro-N-nitroso guanidine (NTG), ethyl methanesulfonate (EMS), ethidium bromide and sodium azide were used as a chemical mutagens to induce mutations in Streptococcus spp. All these mutagenic agents are highly efficient to induce mutations in Streptococcus which has the ability to produce SK with improved activity [16-18]. In addition, it has been observed that production cost of the enzyme by microorganism can be reduced by optimizing the growth conditions and medium components. Regarding the production of SK, different factors played a significant role to increase or decrease the yield of the end product. 
Fermentation conditions including $\mathrm{pH}$, temperature of the fermentation medium, inoculum size, fermentation time, aeration rate, and agitation speed significantly affects the production of SK $[19,20]$. Moreover, carbon source, nitrogen source and selection of substrate and other necessary salts also exert a noteworthy effect on enzyme production [21-23]. Response Surface Methodology is an influential process that is composed of statistical and mathematical skills for planning experiments and analyzing the effect of different factors (independent variables) [24].

The increasing potential of SK administration as a thrombolytic agent triggers the development of quantitative enhancement strategies. Similarly, medium composition and growth parameters are necessary to be optimized for enhanced production of highly active SK. In the present study, indigenously isolated S. agalactiae EBL-20 has been used for enhanced production of highly active SK by EMS induced mutations and optimizing the nutritional requirements as well as culture conditions for the growth of microorganism. Previously cited reports are available demonstrating the enhanced production of SK by inducing mutations in wild Streptococcal strains by physical or chemical means. However, there is no data available presenting the use of $S$. agalactiae for overproduction of SK by inducing mutations and optimization of culture conditions and nutritional requirements. With regards to enhanced SK production; response surface methodology proved to be an efficient attempt.

\section{MATERIAL AND METHODS}

\section{Microorganism and Culture Conditions}

S. agalactiae EBL-20 was previously isolated and identified by API-20-Strep kit (BioMerieux, France) in Enzyme Biotechnology Lab., University of Agriculture, Faisalabad, Pakistan. The culture was maintained on blood agar medium and nutrient agar medium to be used in the present study.

\section{Chemical Mutagenesis of S. Agalactiae Ebl-20 by Ethyl Methanesulfonate (Ems)}

S. agalactiae EBL-20 was exposed to EMS for 0-200 minutes with an interval of 20 minutes to induce mutation. Spore suspension of wild $S$. agalactiae EBL-20 was prepared in tryptone broth which was then washed and suspended in $100 \mathrm{mM}$ potassium phosphate buffer $(\mathrm{pH}$ 7.5). $1 \mathrm{~mL}$ of spore suspension was added aseptically into sterile test tube and introduced with $12 \mu \mathrm{L}$ of EMS [25]. The aliquots were obtained after every 20 minutes, washed and poured on nutrient agar plates after serial dilution. The plates were incubated at $37^{\circ} \mathrm{C}$ for 24 hours. The optimum dose of EMS exposure was selected by formulating 3-log kill curve (Figure 1) and the potent mutant was screened out from the selected colonies by following the procedures described below. 


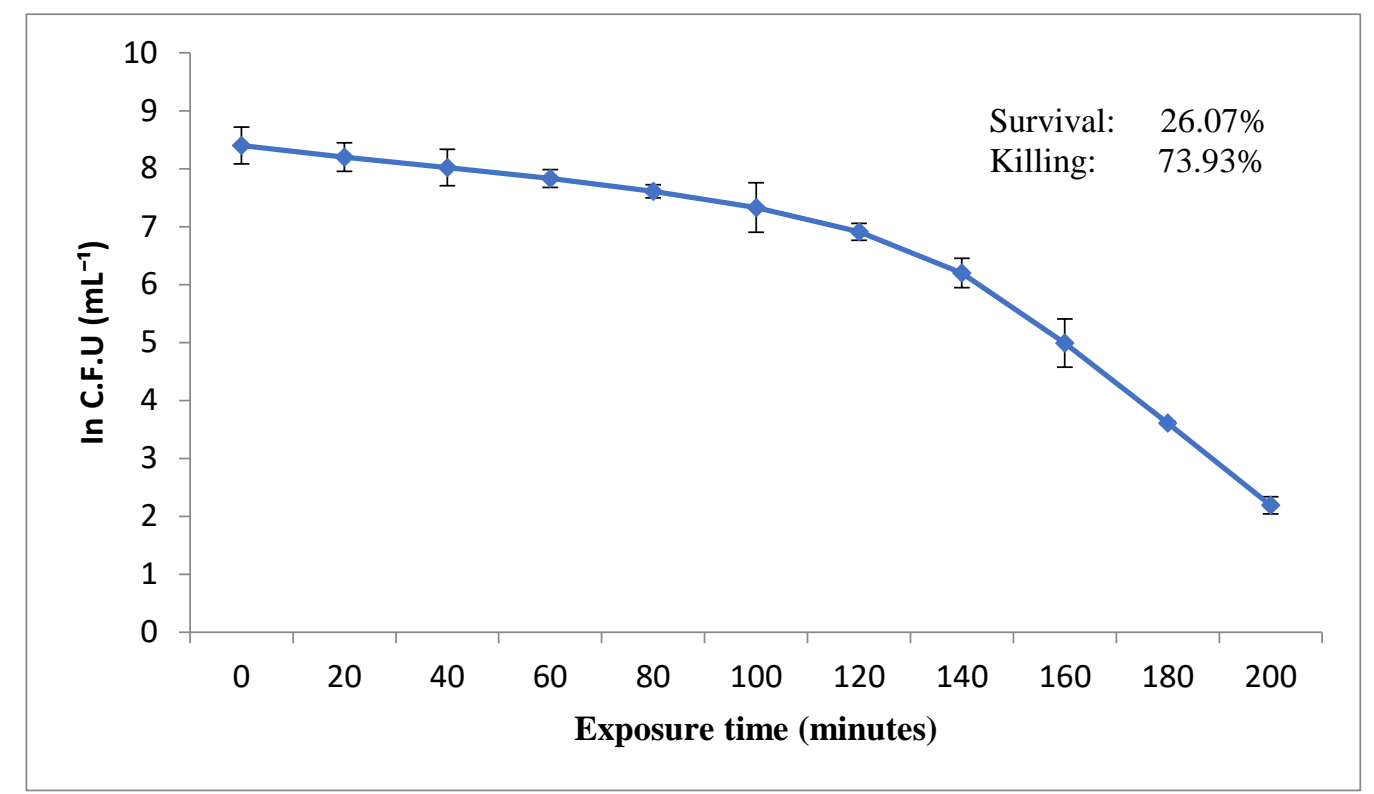

Figure 1 - Formulation of kill curve after mutagenesis of $S$. agalactiae EBL-20 by EMS

\section{Screening of Mutants}

The potent mutated bacterial colonies were screened out by plating bacterial culture on nutrient agar plates supplemented with triton X-100. The colonies grown on triton $X-100$ were further analyzed on skimmed milk agar plates. For this purpose, isolated colonies were allowed to grow on plates containing agarose mixture composed of $0.8 \%$ agarose, $200 \mathrm{uL}$ human plasma, $10 \%$ skimmed milk, $150 \mathrm{mM} \mathrm{NaCl}$ and $50 \mathrm{mM}$ Tris- $\mathrm{HCl}(\mathrm{pH}$ 8.0) [15]. The plates were incubated at $37^{\circ} \mathrm{C}$ for $12-16 \mathrm{~h}$. The activity of $\mathrm{SK}$ was determined by measuring the clear zones around the colonies occurred due to the proteolytic activity of SK [26, 27]. Final selection was accomplished by examining the clot lysis ability of the colonies exhibiting maximum zone of clearance.

The thrombolytic activity of crude enzyme extracted from wild type and mutant derived strain was examined by in vitro blood clot lysis assay [15, 18, 28]. The clot lysis (\%) ability of the selected colonies was determined as:

Clot weight $=$ weight of tube containing clot - weight of empty tube.

Clot lysis $(\%)=($ weight of the lysed clot / weight of the clot before lysis $) \times 100$.

One unit of streptokinase is the concentration of enzyme which converts 1 micro-mole of substrate into product in one minute.

\section{Production of Streptokinase From Mutant Derived Strain}

The colonies of the selected mutant named as $S$. agalactiae EBL-32 grown on nutrient agar plate were picked and inoculated into Todd Hewitt Broth at $37^{\circ} \mathrm{C}$ for $24 \mathrm{~h}$. Liquid state fermentation was performed in triplicate in Erlenmeyer flask containing $50 \mathrm{~mL}$ of preoptimized production media (g/100mL: Glucose- $0.5 \mathrm{~g}$, Yeast Extract- $0.5 \mathrm{~g}, \mathrm{~K}_{2} \mathrm{HPO}_{4}-0.25 \mathrm{~g}$, $\mathrm{KH}_{2} \mathrm{PO}_{4}-0.25 \mathrm{~g}, \mathrm{NaHCO} 3-0.1 \mathrm{~g}$, MgSO $4 \cdot 7 \mathrm{H}_{2} \mathrm{O}-0.04 \mathrm{~g}, \mathrm{CH}_{3} \mathrm{COONa} \cdot 3 \mathrm{H}_{2} \mathrm{O}-0.1 \mathrm{~g}, \mathrm{MnCl}_{2} .4 \mathrm{H}_{2} \mathrm{O}-$ $0.002 \mathrm{~g}$, and $\mathrm{FeSO}_{4} \cdot 7 \mathrm{H}_{2} \mathrm{O}-0.002 \mathrm{~g}, \mathrm{pH} 7.5$ ) [29]. A total of $1 \mathrm{~mL}$ inoculum was introduced into the production media and allowed to grow by liquid state fermentation by incubating at $37^{\circ} \mathrm{C}$ for 24 hours. After incubation the culture was centrifuged at $10,000 \mathrm{~g}$ for 30 minutes to obtain 
the supernatant which was supposed to be the crude enzyme [30]. The nutritional requirements of the microorganism were optimized by analyzing single factor at a time, whereas the fermentation/cultivation conditions were optimized by RSM.

\section{Selection and Optimization of Nutritional Requirements for SK Production}

Effect of different carbon sources (glucose, fructose, sucrose, lactose and starch) on the production of streptokinase by mutant derived $S$. agalactiae EBL-32 was studied. The concentration of the carbon sources was maintained at $0.2 \%, 0.4 \%, 0.6 \%, 0.8 \%$ and $1.0 \%$ while keeping the concentration of all other components constant and activity of SK was monitored [17, 22].

Streptokinase production was analyzed by using different organic nitrogen sources (tryptone, peptone, beef extract, casein and yeast extract) with varied concentrations ranging from $0.2-1.0 \%$ and keeping the concentration of all other components at constant level [19].

Similarly, a variety of substrates including corn steep liquor, sugarcane bagasse, rice polishing and molasses at different concentrations ranging from $0.1-1.0 \%$ were analyzed for the better production of streptokinase from mutant derived $S$. agalactiae EBL-32 [21, 31].

\section{Optimization of Culture Conditions for SK Production}

Four variables including $\mathrm{pH}$, temperature, and incubation/fermentation time and inoculum size were analyzed for their interactive influence on the yield of SK by using a statistical approach. A central composite design (CCD) of response surface methodology (RSM) was employed for five levels of four different variables, and a total of $30\left(=2^{k}+2 k+6\right)$ experimental runs were conducted (Table 1 ), where $k$ is the number of independent factors/variables. The design consisted of sixteen factorial and eight axial experimental runs with six replicates at the centre point to calculate the pure error. All the experiments were performed in triplicate. For each equation term " $X$ ", the second order polynomial coefficient was estimated through multiple regression and the analysis of variance (ANOVA) [32, 33]. Design-Expert 7.0 was used to generate response surfaces and contour plots. 
Table 1 - Central Composite Design (CCD) for the optimization of four process variables for the enhanced production of streptokinase

\begin{tabular}{|c|c|c|c|c|}
\hline Runs & $\mathrm{pH}$ & Temperature $\left({ }^{\circ} \mathrm{C}\right)$ & Incubation time (h) & Inoculum size $(\mathrm{mL})$ \\
\hline 1 & 5 & 25.00 & 72 & 4.00 \\
\hline 2 & 7 & 37.50 & 48 & 3.25 \\
\hline 3 & 7 & 37.50 & 48 & 2.50 \\
\hline 4 & 5 & 50.00 & 72 & 1.00 \\
\hline 5 & 6 & 37.50 & 48 & 2.50 \\
\hline 6 & 7 & 37.50 & 48 & 2.50 \\
\hline 7 & 5 & 25.00 & 72 & 1.00 \\
\hline 8 & 8 & 37.50 & 48 & 2.50 \\
\hline 9 & 5 & 50.00 & 72 & 4.00 \\
\hline 10 & 5 & 50.00 & 24 & 4.00 \\
\hline 11 & 5 & 25.00 & 24 & 1.00 \\
\hline 12 & 7 & 43.75 & 48 & 2.50 \\
\hline 13 & 9 & 50.00 & 24 & 4.00 \\
\hline 14 & 7 & 31.25 & 48 & 2.50 \\
\hline 15 & 9 & 25.00 & 24 & 4.00 \\
\hline 16 & 7 & 37.50 & 48 & 2.50 \\
\hline 17 & 7 & 37.50 & 48 & 2.50 \\
\hline 18 & 9 & 25.00 & 24 & 1.00 \\
\hline 19 & 9 & 50.00 & 72 & 4.00 \\
\hline 20 & 7 & 37.50 & 60 & 2.50 \\
\hline 21 & 9 & 25.00 & 72 & 1.00 \\
\hline 22 & 9 & 25.00 & 72 & 4.00 \\
\hline 23 & 7 & 37.50 & 36 & 2.50 \\
\hline 24 & 5 & 25.00 & 24 & 4.00 \\
\hline 25 & 9 & 50.00 & 72 & 1.00 \\
\hline 26 & 7 & 37.50 & 48 & 1.75 \\
\hline 27 & 7 & 37.50 & 48 & 2.50 \\
\hline 28 & 5 & 50.00 & 24 & 1.00 \\
\hline 29 & 9 & 50.00 & 24 & 1.00 \\
\hline 30 & 7 & 37.50 & 48 & 2.50 \\
\hline
\end{tabular}

\section{Determination of Streptokinase Activity}

Activity of streptokinase was determined by using casein digestion method in which liberated tyrosine was determined from digested casein after plasminogen activation [33]. Reaction mixture (2 mL) was prepared by adding $10 \mathrm{mg}$ casein, $50 \mathrm{mM} \mathrm{Tris-} \mathrm{HCl}(\mathrm{pH} 8.0)$ to $0.1 \mathrm{ml}$ of supernatant. The reaction process was carried out at $37^{\circ} \mathrm{C}$ for 20 minutes and the reaction mixtures were kept on ice for 30 minutes after terminating the reaction by adding $2.6 \mathrm{~mL}$ of $5 \%$ trichloroacetic acid (TCA) and $0.4 \mathrm{~mL}$ of $3.3 \mathrm{M} \mathrm{HCl}$. The mixture was then 
filtered using Whatman paper and absorbance was measured at $280 \mathrm{~nm}$. A standard SK curve was plotted to determine the activity of streptokinase [34].

\section{Statistical Analysis}

Two factorial complete randomized design (CRD) was used for the optimization of nutritional requirements of the bacterial strain. Data obtained for the optimization of four process variables by RSM was used to determine the regression coefficients of the second-order multiple regression models.

$Y_{\text {yield }}=b o+\sum_{i=1}^{k} b i X i+\sum_{i=1}^{k} b i i X i^{2}+\sum_{i>j}^{k} \sum_{j}^{k} b i j X i X j+e$ where, Yyield is the predicted response variable (SK activity); $k$ is the number of variables being considered in the experiment; $b_{0}, b_{i}, b_{i i}$, and $b_{i j}$ are regression coefficients and $e$ is the random error.

\section{RESULTS AND DISCUSSION}

Although a number of plasminogen activators are available for thrombolytic therapy, streptokinase has widely been used in developing countries mainly due to its high effectiveness and low cost. It is used as a thrombolytic agent for AMI in about half of the countries worldwide where patients do not have rapid access to catheterization [35]. Due to its wide application in cardiovascular diseases and their outcomes, there is an urge for reduction in its production cost. Native SK can easily be produced inexpensively by optimizing the production process of fermentation [15, 36]. The comparison of our findings with previous studies shows that strain improvement can be achieved by exposing the wild Streptococcus spp. to certain mutagenic agents and one advantage of using customary procedures of inducing mutations by using different mutagens is the cost effectiveness.

\section{Mutagenesis}

The study was conducted to enhance the yield of SK from S. agalactiae by exposing the wild strain to EMS. The kill curve was formulated using colony forming units and 180 minutes exposure time was selected as the optimum dose where percentage survival and killing of bacterial strain was $26.07 \%$ and $73.93 \%$, respectively (Figure 1 ). The colonies showing restriction against Triton X-100 were analyzed for SK production. High SK producing colonies of $S$. agalactiae were screened out by Casein-plasminogen overlay and percentage clot lysis methods, respectively. The zone of clearance of selected colonies was determined on skimmed milk agar plates and the colonies showing maximum zone of clearance (named as EMS-1 to EMS-11) were considered as the best mutants and further subjected to clot lysis assay as shown in Table 2. It was analyzed that the activity of streptokinase shown by EMS-5 was highest with $8.2 \mathrm{~mm}$ zone size (Figure 2) and 54.37\% ability of clot lysis. The selected strain was named as $S$. agalactiae EBL-32 which was further exploited for enhanced production of SK by optimizing the conditions. Wild $S$. agalactiae EBL-20 exhibited a zone size of $4 \mathrm{~mm}$ (Figure 2) with $32.65 \%$ clot lysis. Strain improvement study resulted into 1.6 fold increased production of SK from mutant derived $S$. agalactiae EBL-32 as compared to the wild strain. This result revealed that chemical mutagenesis of wild bacterial strain increases the yield of end product. One of the previous findings demonstrated that SK yield from S. equismilis mutated by UV radiations was $120 \%$ more than that of the wild strain and the SK yield from S. equisimilis exposed to NTG was $146 \%$ 
more as compared to the wild strain [16]. In another study, 3 fold increased production of SK was reported from $S$. equisimilis by exposing the wild strain to ultraviolet radiations [17].

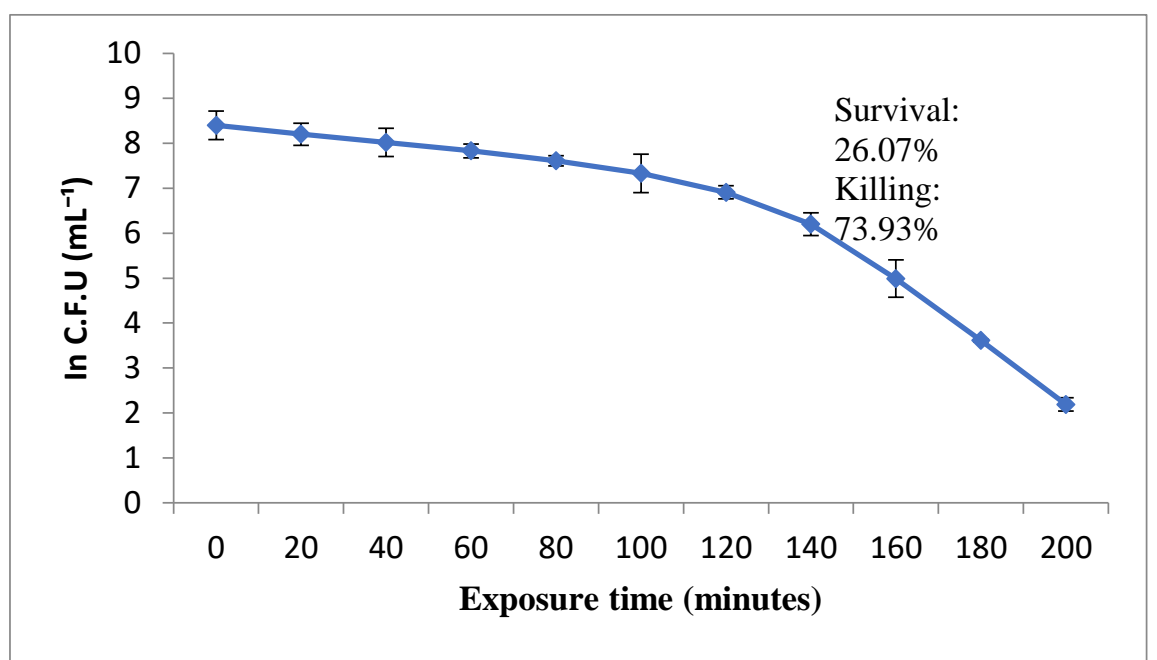

Figure 2 - Formulation of kill curve after mutagenesis of $S$. agalactiae EBL-20 by EMS

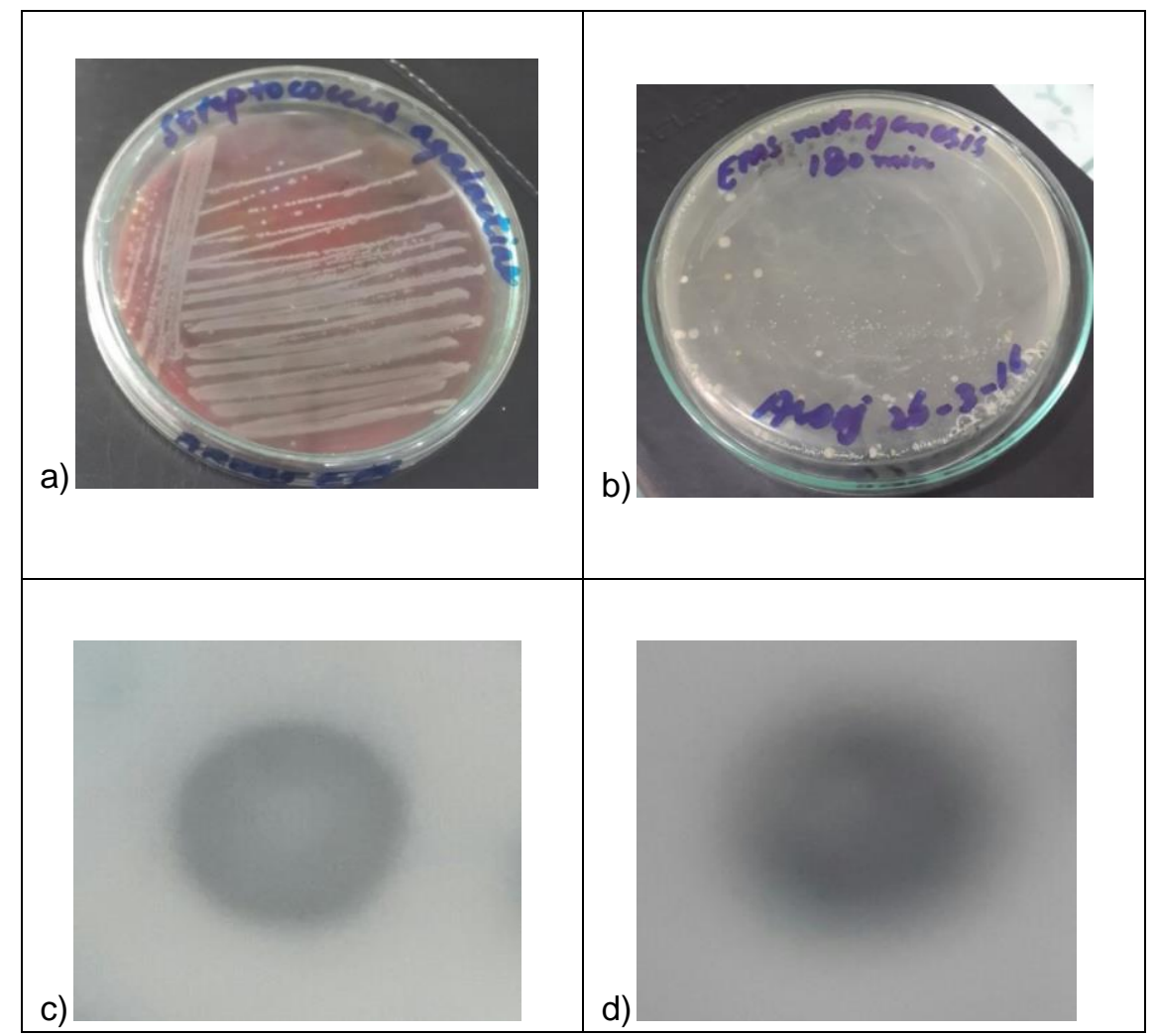

Figure 2 (a): Culture of $S$. agalactiae EBL-20 maintained on blood agar media, (b) Selected plate after exposure to EMS, (c and d) maximum zone of casein hydrolysis observed by wild $S$. agalactiae EBL-20 (4 mm) and mutant derived $S$. agalactiae EBL-32 $(8.2 \mathrm{~mm})$, respectively. 
Table 2 - Measurements of zone size and clot lysis (\%) ability of selected colonies after exposure to EMS

\begin{tabular}{|c|c|c|}
\hline $\begin{array}{l}\text { S. agalactiae } \\
\text { strains }\end{array}$ & $\begin{array}{l}\text { Zone } \\
(\mathrm{mm})\end{array}$ & $\begin{array}{l}\text { Clot lysis } \\
\text { (\%) }\end{array}$ \\
\hline Wild/control & 4.0 & 32.65 \\
\hline EMS-1 & 5.8 & 36.83 \\
\hline EMS-2 & 6.5 & 39.74 \\
\hline EMS-3 & 5.9 & 34.11 \\
\hline EMS-4 & 6 & 36.33 \\
\hline EMS-5 & 8.2 & 54.37 \\
\hline EMS-6 & 7.9 & 47.63 \\
\hline EMS-7 & 6.5 & 35.43 \\
\hline EMS-8 & 7.0 & 40.54 \\
\hline EMS-9 & 7.0 & 39.26 \\
\hline EMS-10 & 7.3 & 41.28 \\
\hline EMS-11 & 7.1 & 40.22 \\
\hline
\end{tabular}

\section{Selection and optimization of carbon and nitrogen source and substrate}

A noteworthy increase in enzyme production can be obtained by optimizing the nutritional requirements of the microorganism. The present study describes the optimum concentrations of substrate, carbon and nitrogen sources for enhanced SK production mutant derived strain of $S$. agalactiae. Among the different carbon sources, the mutant derived S. agalactiae EBL-32 showed the best SK activity of $93.54 \mathrm{UmL}^{-1}$ with $0.6 \%$ glucose. The production of SK was not promoted by using lactose and starch in the medium; however the production was satisfactory by using $0.4 \%$ fructose (Figure $3 a$ ). Regarding the nitrogen source, the improved production of SK was observed by using peptone $(0.6 \%)$ in the fermentation medium showing $95.43 \mathrm{UmL}^{-1}$ SK activity (Figure 3b). These results demonstrate that carbon and nitrogen sources played a significant role in obtaining high yield of SK ${ }^{5}$. Previous findings suggested glucose as the best carbon source for SK production from Streptococcus spp. It has been reported that $0.25 \%$ glucose and $1.5 \%$ casein are the optimum concentrations of carbon and nitrogen sources, respectively for better growth of $S$. pyogenes and $S$. equisimilis [22]. In comparison, our findings revealed that peptone is the best organic nitrogen source for growth of mutant derived $S$. agalactiae EBL-32. However, the growth was found to be stable in the presence of yeast extract and typtone but it was decreased in the presence of beef extract. In addition, maximum SK Brazilian Archives of Biology and Technology. Vol.62: e19170813, 2019- www.scielo.br/babt 
activity (96.21 $\mathrm{UmL}^{-1}$ ) was observed by adding $0.2 \% \mathrm{CSL}$ in the production medium. However, the production of SK was found to be lowered in the presence of rice polishing and sugarcane bagasse (Figure 3c). CSL exerts a positive effect on the production of SK $[20,21]$. Analysis of variance revealed the significant impact of all the nutritional sources with $p<0.01$ (as given in supplementary Table S1-S3).
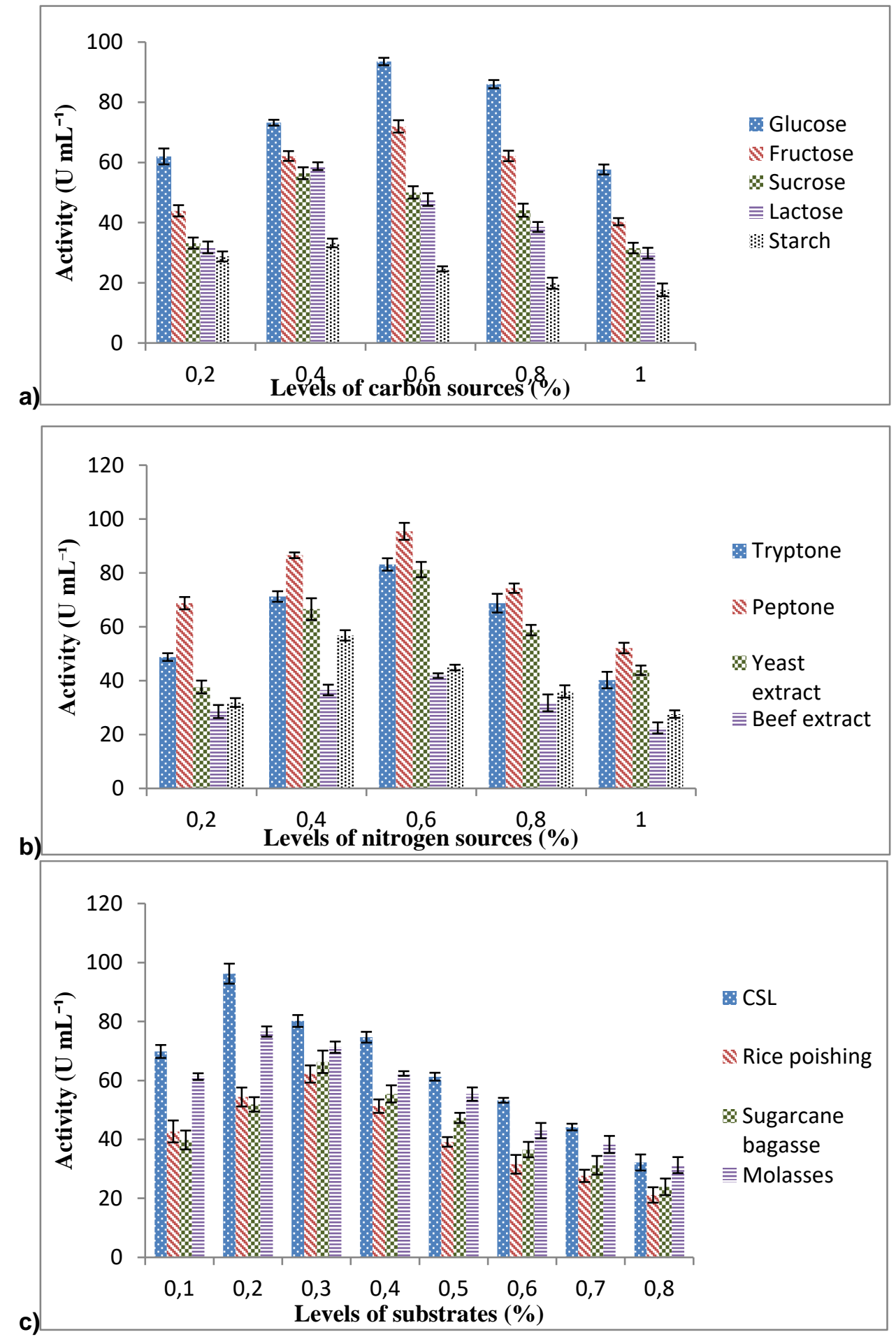

Figure 3 - Selection and optimization of (a) carbon source, (b) nitrogen source and (c) substrate for the production of SK from mutant derived S. agalactiae EBL-32. Optimization of SK production by experimental designs and RSM 
Analysis of variance (ANOVA) of the data obtained for second order response surface model is presented in Table 3 . The high $F$ value, $F_{\text {model }}=860.66$ indicates that complete quadratic regression model is significant; whereas, the non-significant lack of fit shows that second-order model is a satisfactory approximation to the exact response surface. Adjusted coefficient of determination $\left(R_{a d j}^{2}\right)$ evaluated the peculiarity of fit of the polynomial model equation. For mutant derived $S$. agalactiae EBL-32, linear terms; incubation time and $\mathrm{pH}$, whereas, quadratic terms; $\mathrm{pH}$ and incubation time significantly contributed to the fitted model. The significance of $R_{a d j}^{2}=0.9976$ indicates that $99 \%$ variation in response has been explained by the terms involved in the quadratic model [37].

The statistical signification of the fitted model of parameters being studied for response (SK yield) was evaluated by the $F$ ratio and its equivalent $p$ values; mentioned in Table 3. The $p$ value indicates the relative significance of the terms involved with the particular parameter and analyzes the significance of coefficients. The smaller $p$ value for a parameter indicates that there is more influence of the observed parameter [38]. It was observed that the first order interactive influence of all the analyzed variables is significant $(p<0.0001)$ with the non-significant interactive effect of temperature and incubation time. For valid application of Analysis of Variance, residuals of the model must be normally distributed. Figure 4 shows the normal probability plot for residuals; normality of residuals is substantiated by the points in close proximity to the straight line.

Table 3 - Analysis of variance for the quadratic model for mutant derived S. agalactiae EBL-32

\begin{tabular}{|l|l|l|l|l|l|}
\hline Source of variation & $\begin{array}{l}\text { Sum of } \\
\text { Squares }\end{array}$ & df & $\begin{array}{l}\text { Mean } \\
\text { Square }\end{array}$ & $\begin{array}{l}\text { F } \\
\text { Value }\end{array}$ & $\boldsymbol{p}$-value \\
\hline Model & 36426.85 & 14 & 2601.92 & 860.66 & $<0.0001$ \\
Residual & 45.35 & 15 & 3.02 & & \\
Lack of Fit & 37.73 & 10 & 3.77 & 2.48 & 0.1644 \\
Pure Error & 7.62 & 5 & 1.52 & & \\
Cor Total & 36472.19 & 29 & & & \\
\hline
\end{tabular}




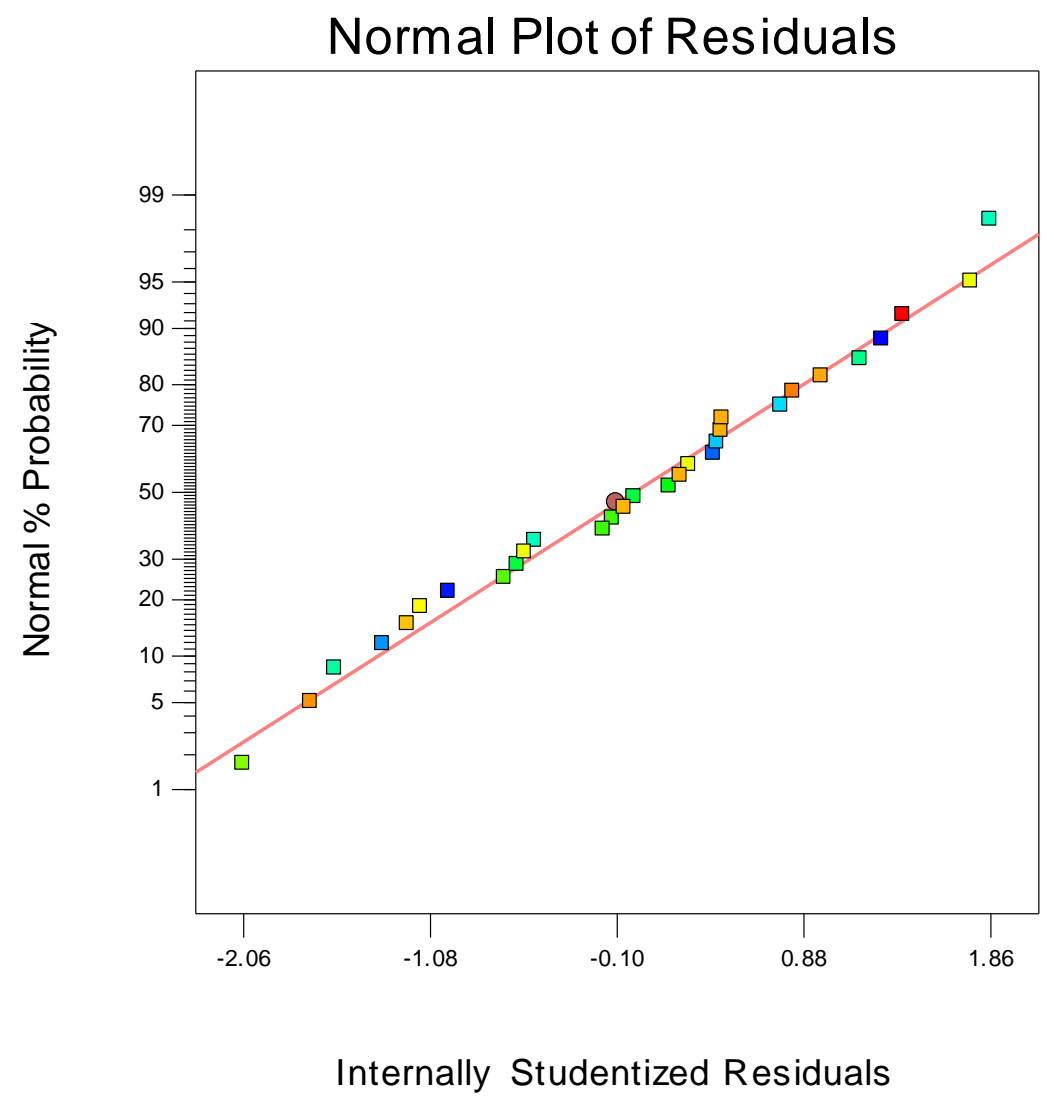

Figure 4 - Normal probability plot of residuals

\section{Response surface analysis}

Using the design of experimental data, the second degree polynomial model for SK yield from mutant derived $S$. agalactiae EBL-32 was obtained (in terms of actual factors) as: $Y=-862.59+242.43 X_{1}+22.41 X_{2}-10.69 X_{3}+8.38 X_{4}-0.12 X_{1} X_{2}+0.17 X_{1} X_{3}-0.80 X_{1} X_{4}-$ $5.58 X_{2} X_{3}-0.23 X_{2} X_{4}+0.05 X_{3} X_{4}-17.16564 X_{1}^{2}-0.28008 X_{2}^{2}+0.09 X_{3}^{2}-1.05891 X_{4}^{2}$ where $\mathrm{Y}$ is the response variable for $\mathrm{S}$. agalactiae $\mathrm{EBL}-32 ; \mathrm{X} 1, \mathrm{X} 2, \mathrm{X} 3$, and $\mathrm{X} 4$ are the actual factors, namely, $\mathrm{pH}$, temperature, incubation time and inoculum size, respectively.

3-D surface plots were generated by considering an infinite number of combinations of two test variables simultaneously, while keeping other two variables constant. The predictor variables are displayed on the $x$ - and $y$-scales, and the response variable is represented by a smooth surface. Figure 5 represents the surface plots for two variables keeping other variables at specified levels. Curvature in the surface plots indicates the interaction between variables holding other variables constant at some pre-specified level.

A very strong interaction can be seen between temperature and $\mathrm{pH}$ for the growth of S. agalactiae EBL-32 which is indicated by the curvature of the plots (Figure 5a). It was observed that SK activity increases as the $\mathrm{pH}$ and temperature of the culture medium increases and maximum SK yield was obtained from both the strains at $\mathrm{pH} 7$ and temperature $37.5^{\circ} \mathrm{C}$ while by a further increase in both the parameters, there was a gradual decline in SK activity. This may be due to the decline in $\mathrm{pH}$ of the fermentation medium over 
time by the production of acidic products at the end of the metabolism. Degradation of SK usually takes place at high temperature as this fibrinolytic enzyme is active between the temperature range of $27-45^{\circ} \mathrm{C}$ [39]. In addition, it was observed that there is a moderate interaction between $\mathrm{pH}$ and inoculum size, while keeping the other two variables constant (Figure 5b). Figure $5 \mathrm{c}$ indicates a significant interaction between $\mathrm{pH}$ and inoculum size and shows that SK activity is increased by increasing the inoculum size upto $2.50 \mathrm{~mL}$ at $\mathrm{pH} 7$ but a decreased activity of SK was observed by further increase in the volume of inoculum as well as $\mathrm{pH}$ of the culture medium. An increased SK yield was obtained by increasing the incubation time and temperature gradually, revealing a noteworthy interaction between the two variables. In addition a sudden decreased SK activity was observed at lower temperatures (Figure $5 \mathrm{~d}$ ). No significant interaction was observed between temperature and incubation time, but the two variables have a strong individual affect on the yield of SK (Figure 5e). Figure $5 f$ interprets that incubation time and inoculum size acts as a function of SK productivity, while keeping the remaining two variables constant. However, inoculum size exerts a negative effect on SK productivity [20].

Based on the statistical results, the maximum production of streptokinase with $168.46 \mathrm{U} \mathrm{mL}^{-1}$ from mutant derived $S$. agalactiae EBL-32 was obtained at $\mathrm{pH}$ 7; temperature $37.5^{\circ} \mathrm{C}$; incubation time 36 hours and inoculum size $2.50 \mathrm{~mL}$ as presented in the figures. 

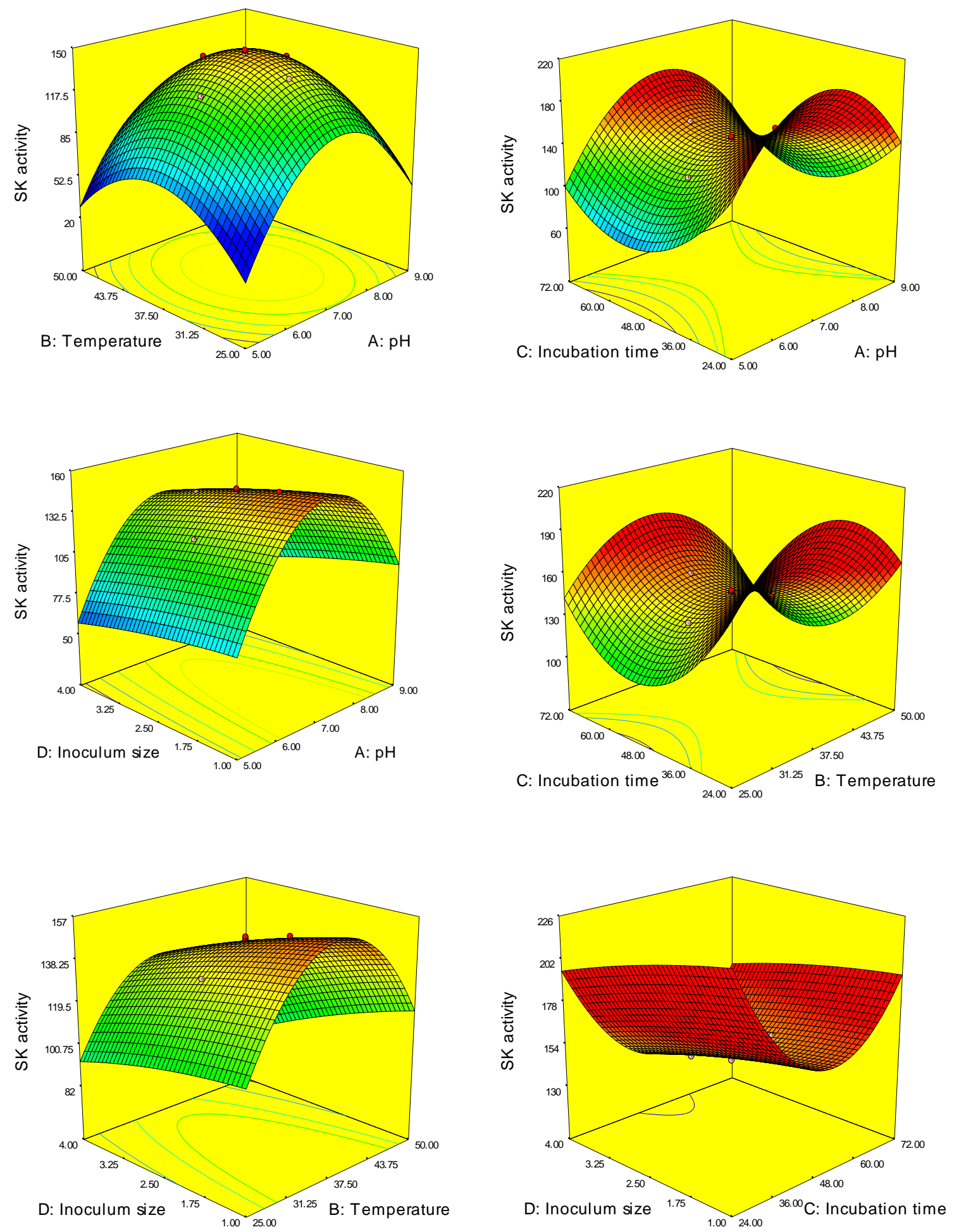

Figure 5 - 3-D surface plots for the production of SK from mutant derived S. agalactiae EBL-32.

After the optimization studies, enhanced production of streptokinase was observed from mutant derived $S$. agalactiae EBL-32 with $168.46 \mathrm{UmL}^{-1}$ activity suggesting that mutating the wild bacterial strain by EMS is an effective technique to obtain over-production of SK. The optimization study by RSM concludes that production of SK from mutant derived S. agalactiae EBL-32 was increased by 1.92 fold after optimization of culture conditions and 
nutritional requirements. Based on the literature cited, $110 \%$ increase in SK yield was observed after optimizing the culture conditions and nutritional requirements [19].

\section{CONCLUSION}

The study revealed that induction of random mutations in the genome of microorganism is an efficient technique to improve the production of streptokinase. The most remarkable achievement of this study was the highly potent mutant $S$. agalactiae EBL-32 and enhanced production of streptokinase by fermentation process. These results suggest that EMS is an effective mutagen for strain improvement of $S$. agalactiae EBL-20. Moreover, optimization studies could be the better option to make it economically feasible for the treatment of poor heart patients in developing countries. Hence, the study concludes that $S$. agalactiae can be exploited for large scale production of streptokinase. Although thrombolytic therapy by using native streptokinase is useful; however its administration provokes allergic reactions. In future, more focus should be made for the production of genetically modified streptokinase to extend its half life in blood circulation and reducing immunogenicity.

Funding: The research was funded by Higher Education Commission (HEC), Pakistan [NRPU project\# 4921]

Conflicts of Interest: The authors declare no conflict of interest. The funders had no role in the design of the study; in the collection, analyses, or interpretation of data; in the writing of the manuscript, or in the decision to publish the results.

\section{REFERENCES}

1. Collen D, Stump DC, Gold HK. Thrombolytic therapy. Annu Rev Med. 1998; 39: 405-423.

2. Furie B, Furie BC. Mechanisms of thrombus formation. N Engl J Med. 2008; 359: 938-949.

3. Iqbal O, Tobu M, Demir M, Fareed J, Aziz S, Messmore H. The Role of Thrombolytic Drugs in the Management of Acute Myocardial Infarction and Stroke. Turk J Haematol. 2002; 19: 151-177.

4. Francis CW, Marder VJ. Fibrinolytic therapy for venous thrombosis. Prog Cardiovasc Dis. 1991; 34: 193-204.

5. Shilpi B, Bhattacharya S, Das A, Palaniswamy M, Angayarkanni J. Application of Multifactorial Experimental Design for Optimization of Streptokinase Production Using Streptococcus equisimilis SK-6. Arab J Sci Engg. 2017; 42: 2273-2277.

6. Ghosh M, Pulicherla KK, Rekha VPB, Rao GV, Sambasiva KRS. Int J Pharm Pharm Sci. 2012; 4: 38-42.

7. Brockway WJ, Castellino FJ. A characterization of native streptokinase and altered streptokinase isolated from a human plasminogen activator complex. Biochem. 1974; 13: 2063-2070.

8. Babashamsi BM, Razavian MH, Nejadmoghaddam MR. Production and purification of streptokinase by protected affinity chromatography. Avicenna J Med Biotechnol 2009; 1: 47 51.

9. Johnsen, L.B., K. Poulsen, M. Kilian and T.E. Petersen. 1999. Purification and cloning of a streptokinase from Streptococcus uberis. Infect. Immun. 67: 1072-1078. 
10. Zhang, X.W., T. Sun, X.N. Huang, X. Liu, D.X. Gu, and Z.Q. Tang. Recombinant streptokinase production by fed-batchcultivation of Escherichia coli. Enzyme Microbiol Technol. 1999; 24: 647- 50.

11. Kunamneni A, Abdelghani TTA, Ellaiah P. Streptokinase--the drug of choice for thrombolytic therapy. J Thromb Thrombolysis. 2007; 23: 9-23.

12. Madhuri DH, Manohar M, Neha AS, Mohanasrinivasan V, Subathra DC. Studies on isolation, screening and strain improvement of streptokinase producing $\beta$ - hemolytic streptococci. World J Sci Tech. 2011; 1: 7-11.

13. Kumar PS, Pulicherla KK, Sambasiva KRS. Current Status of Production, Clinical Usage and Market Scenario of Streptokinase. J Pronline. 2012; 5: 4223-4229.

14. Bhardwaj S, Angayarkanni J. Streptokinase production from Streptococcus dysgalactiae subsp. equisimilis SK-6 in the presence of surfactants, growth factors and trace elements. Biotech. 2015; 5: 187-193.

15. Babu V, Devi S. Exploring the in vitro thrombolytic potential of streptokinase-producing $\beta$ hemolytic Streptococci isolated from bovine milk. J Gen Appl Microbiol. 2015; 61: 139-146.

16. Abdelghani TTA, Kunamneni A, Ellaiah P. Isolation and mutagenesis of streptokinase producing bacteria. Am J Immunol. 2005; 1: 125-129.

17. Devi CS, Mohanasrinivasan V, Vaishnavi B, Selvarajan E, Naine SJ. Optimization Studies for Enhanced Production of Streptokinase by Streptococcus equisimilis UVM6. J Pure \& Appl Microbiol. 2013; 7: $1-5$.

18. Gull-E-Faran, Zia MA, Shahid M, Abdullah S. Improved Streptokinase production; UV irradiation of Streptococcus equisimilis. Professional Med J. 2015; 22: 656-663.

19. Vellanki RN, Potumarthi R, Mangamoori LN. Constitutive expression and optimization of nutrients for streptokinase production by Pichia pastoris using statistical methods. Appl Biochem Biotechnol. 2009; 158: 25-40.

20. Patel VP, Patel KM, Patel RM. Isolation and optimization of streptokinase production by batch fermentation. Int J Pharm Biol Archives. 2011; 2: 1488-1492.

21. Zia MA, Faisal, R, Abbas RZ, Gull-e-Faran, Saleemi MK, Khan JA. Comparison of Streptokinase activity from Streptococcus mutans using different substrates. Pak Vet J. 2013; 33: 77-79.

22. Elhalafawy KA, Guirgis AA, Elkousy SM, Rizk NM, Eldourghamy AS. Monitoring streptokinase and some factors that affect its production during growth curve of two streptococcal strains. Sci J Fac Sci. 2009; 23: 85-103.

23. Lee HS. How safe is the readministration of streptokinase. Drug saf. 1995; 13: 76-80.

24. Myers RH, Montgomery DC. "Response surface methodology," in Process and Product Optimization Using Designed Experiments. Wiley-Interscience, New York, USA; 1995.

25. Ibrahim SA, Sullivan DJO. Use of Chemical Mutagenesis for the Isolation of Food Grade $\beta$ Galactosidase Overproducing Mutants of Bifidobacteria, Lactobacilli and Streptococcus thermophilus. J Dairy Sci. 2000; 83: 923-930.

26. Ozegowski JH, Gerlach D, Kohler W. Influence of physical parameters on the production of streptococcal extracellular proteins in cultures with stabilized $\mathrm{pH}$ : 2. Temperature dependence of extracellular protein production. Zentralbl Bakteriol Mikrobiol Hyg A. 1983; 254: 361-369.

Brazilian Archives of Biology and Technology. Vol.62: e19170813, 2019- www.scielo.br/babt 
27. Sahni G, Kumar R, Roy, C, Rajagopal K, Nihalani D, Sundaram V. et al. Clot specific streptokinase proteins processing altered plasminogen activation characteristics and a process for their preparation.US Patent No. 7,163,817, B2; 2007.

28. Holmstrom, B. Streptokinase assay on large agar diffusion plates. Acta Chem Scand 1965; 19: $1549-1554$.

29. Baewald G, Mayer G, Heikel R, Volzke KD, Roehlig R, Decker KL, Koehler W, Gerlach D. Fermentative production of Streptococcus metabolites, especially streptokinase. German patent DD 111096, 1975.

30. Hamid M, khalil-ur-rehman, Asghar M, Sarwar M. Streptokinase as Fibrinolytic Agent. Asian J Chem. 2012; 24: 1809-1814.

31. Ghaffar A, Ahmed B, Munir B, Faisal R, Mahmood Z. Production and characterization of streptokinase enzyme by using Streptococcus mutans strain in liquid state fermentation through corn steep liquor (CSL) substrate. Biochem Physiol. 4, 178. doi: 10.4172/21689652.1000178. 2015.

32. Khuri Al, Cornell JA. Response Surfaces: Design and Analysis, Marcel Dekker, New York, USA; 1987.

33. Mounter L.A, Shipley BA. The inhibition of plasmin by toxic phosphorus compounds. J Biol Chem. 1957; 231: 855-861.

34. El-Mongy MA, Taha TM. In vitro detection and optimization of streptokinase production by two streptococcal strains in a relatively low cost growth medium. Intern Res J Microb. 2012; 3: 153-163.

35. Raee MJ, Ghasemian A, Maghami S, Ghoshoon MB, Ghasemi Y. Cloning, purification and enzymatic assay of streptokinase gene from Streptococcus pyogenes in Escherichia coli. Minerva Biotechnol. 2017; 29: 8-13.

36. Banerjee A, Chisti Y, Banerjee UC. Streptokinase, a clinically useful thrombolytic agent. Biotechnol Adv. 2004; 22: 287-307.

37. Box GEP, Hunter WG, Hunter JS. Statistics for Experimenters, John Wiley \& Sons, New York, USA; 1978.

38. Montgomery D.C. Design and Analysis of Experiments, John Wiley \& Sons, New York, USA; 2001

39. Dubey R, Kumar J, Agrawala D, Char T, Pusp P. Isolation, production, purification, assay and characterization of fibrinolytic enzymes (Nattokinase, Streptokinase and urokinase) from bacterial sources. Afr J Biotechnol. 2011; 10: 1408-1420.

(ㅇ) $\mathbb{Q}$ (c) 2018 by the authors. Submitted for possible open access publication under the terms and conditions of the Creative Commons Attribution (CC BY NC) license (http://creativecommons.org/licenses/by/4.0/).

\footnotetext{
*Correspondence: elegant_arooj@hotmail.com; Tel.: +92-41-9200161
} 